\title{
Photoacoustic characterization of transient defects in potassium dihydrogen phosphate crystals
}

\author{
O. Martínez Matos, ${ }^{1}$ G. A. Torchia, ${ }^{1,2}$ G. M. Bilmes, ${ }^{1,3}$ and J. O. Tocho ${ }^{1,2}$ \\ ${ }^{1}$ Centro de Investigaciones Opticas-CIOp- (CONICET - CIC), CC 124, 1900 La Plata, Argentina \\ ${ }^{2}$ Facultad de Ciencias Exactas, Universidad Nacional de La Plata, 47 y 115, 1900 La Plata, Argentina \\ ${ }^{3}$ Facultad de Ingeniería, Universidad Nacional de La Plata, 1 y 47, 1900 La Plata, Argentina \\ (Received 27 June 2003; revised manuscript received 13 February 2004; published 3 June 2004)
}

\begin{abstract}
Transient defects in potassium dihydrogen phosphate (KDP) were characterized by using the acoustic signals generated in the crystal when it is impinged with pulsed laser radiation. These defects are produced by simultaneous absorption of two $\lambda=266 \mathrm{~nm}$ photons and they show linear absorption in the visible and UV spectral region. The decay kinetics of the defects has been studied by a new method based on the analysis of the acoustic signal generated by visible pulses. The acoustic measurement of the decay time shows a nonexponential decay and it is free from thermal lensing or beam deformation by other causes, effects that can alter the pure optical measurements. We propose that the origin of the photoacoustic signal is the heat released by the deexcitation of the energy levels of the defects when they are excited by visible pulses. This mechanism, optical absorption and nonradiative relaxation of defects, could be the reason for some depletion in the yield of several devices based on KDP. This phenomena must be carefully taken in account, when KDP crystals are used in combination with Nd:YAG (YAG, yttrium aluminum garnet) lasers for second-harmonic generation from $\lambda=532 \mathrm{~nm}$ to $\lambda=266 \mathrm{~nm}$.
\end{abstract}

DOI: $10.1103 /$ PhysRevB.69.224102

PACS number(s): $61.80 .-\mathrm{x}, 42.65 . \mathrm{Ky}, 77.84 . \mathrm{Fa}$

\section{INTRODUCTION}

Potassium dihydrogen phosphate(KDP) crystals are probably the most extensively used material in nonlinear optics because of its extended applications as second-harmonic generators, optical switches, parametric oscillators, or optical parametric amplifiers.

Over the past decades, it has been studied transient defects (TR defects) generated in KDP crystals by $X$-radiation, ${ }^{1-3} \gamma$-radiation, ${ }^{4,5}$ particle electron bombardment, ${ }^{6,7}$ and UV $(\lambda=266 \mathrm{~nm}, \lambda=355 \mathrm{~nm})$ radiation. ${ }^{3,8-10}$ The optical absorption spectra of TR defects measured immediately after the irradiation or bombardment have a broad band spectra in the region from 1.5 to $5.5 \mathrm{eV}$. TR defects in KDP absorb light very efficiently all over the visible spectrum. They are not permanent and they can be bleached by irradiation or heating. The decay of the transient absorption is not a single exponential but details of its kinetics are not well known.

For UV irradiation, it is accepted that TR defects are produced by two-photon absorption when an electron-hole pair is produced in the bands of the material. The pair relaxes partially and electron-hole can be stabilized in a couple of positive and negative traps as it is common for color centers in alkaline halides.

Still now there is not a definitive identification of the nature of the traps but it is generally accepted the involvement of the $\mathrm{PO}_{4} \mathrm{H}_{2}^{-}$group in the optical active center/s. Pump and probe Raman experiments have shown that the intensity of the $\mathrm{PO}_{4}$ vibrational mode at $915 \mathrm{~cm}^{-1}$ is increased when defects are present. ${ }^{10}$ At least three TR-defects with these characteristics were identified by electron paramagnetic resonance (EPR), KDP and $\mathrm{NH}_{4} \mathrm{H}_{2} \mathrm{PO}_{4}$ (ADP): the $A$ radical $\left(\mathrm{PO}_{4} \mathrm{H}^{-}\right)$representing a hole localized at one oxygen near a proton vacancy in the basic unit; the $B$ radical $\left(\mathrm{PO}_{4} \mathrm{H}_{2}\right)$ that is a self-trapped hole at the basic unit, and the $D$ radical $\left(\mathrm{PO}_{4} \mathrm{H}_{3}^{-}\right)$that is a neutral hydrogen $\mathrm{H}^{0}$ localized near the basic group. The $A$ and $B$ radicals represent hole traps while the $D$ radical is an electron trap. ${ }^{7}$ Other electron trap produced during UV irradiation at low temperature is the neutral hydrogen $\mathrm{H}^{0}$ itself that can be considered the complementary defect of the $A$ radical. At $77 \mathrm{~K}$, irradiation of KDP produces a concentration of $\mathrm{A}$ radicals and $\mathrm{H}^{0}$ centers as high as $1018 \mathrm{~cm}^{-3}$. $^{3}$

It is well known from the literature that the analysis of the photoacoustic signals generated by pulsed laser excitation can be used as a sensitive and accurate technique to study several physical phenomena in solids. For example, phase transitions ${ }^{11-14}$ and luminescent quantum efficiencies, ${ }^{15,16}$ were determined in this way.

In this line, Tocho et al. ${ }^{17}$ have explored the phase matching conversion from $532 \mathrm{~nm}$ to $266 \mathrm{~nm}$ in KDP crystals by the photoacoustic technique. That work concluded that the amount of heat produced by the absorption of linear and nonlinear UV radiation by the crystal could not explain completely the photoacoustic signal generated in their experiments. The authors suggest that TR defects generated during irradiation could contribute to an essential part of the photoacoustic signal when these crystals are impinged with nanosecond-laser pulses in the visible and UV spectrum. TR defects absorb light increasing the local temperature of the illuminated volume. The surface of this volume suffers an expansion and contraction by means of the thermoelastic effect, generating photoacoustic signal.

Apart from point defects, other mechanism for the generation of acoustic signals in irradiated KDP must be considered. As it was demonstrated in $\mathrm{NaCl}$ and $\mathrm{SiO}_{2}$ samples, strong acoustic signals can be produced by generating free electrons and, subsequently, heating them via free electronphoton-lattice interaction. ${ }^{18}$ In a two wavelength experiment, 
where one wavelength generates the free carriers and the other pulse produces the lattice heating, the acoustic signal occurs only for very short delay times, no more than the pulse duration because the lifetimes of free carriers are extremely short (picosecond). On the other hand, as the lifetime of TR defects occurring in KDP are quite much longer, acoustic signals produced by absorption of TR defects could be generated for longer delays than picosecond.

The present work is aimed to go deeper in a physical explanation of the origin of the photoacoustic signal generated when KDP crystals are irradiated by UV, visible, or both types of pulsed radiation. Also, we explore the capability of the photoacoustic methods for the study of TR defects generated in KDP. Better knowledge of these aspects are very important in technological applications because they are connected with the efficiency of nonlinear processes that lead to UV generation and with the optical performance of KDP when these crystals are used directly exposed to UV radiation of high power. We explore the TR defects by using the acoustic signal after UV or visible absorption. As it will be discussed in this work, the photoacoustic technique can be used to get complementary information in relation to experiments performed with conventional optical techniques.

\section{EXPERIMENT}

Experiments were performed with two different KDP crystals. Sample 1, a cube of $1 \mathrm{~cm}^{3}$ size, was a very old one manufactured by Lasermetrics Inc., ${ }^{19}$ in the early eighties for doubling $\lambda=600 \mathrm{~nm}$ radiation in a phase matching type-I arrangement. Sample 2 was a fresh one grown at Moscow, Russia in 2000. It had a size of $1 \times 1 \times 0.5 \mathrm{~cm}^{3}$ and it was cut for second harmonic generation of $1060 \mathrm{~nm}$. No substantial differences were found in the experiments performed with the two samples. Thereafter we present the data corresponding to sample 2. In the experimental arrangement used in this work, laser pulses (10 ns) of a Nd: YAG(YAG, yttrium aluminum garnet) $Q$-switch laser converted to the second harmonic $(\lambda=532 \mathrm{~nm})$ and to the fourth harmonic $(\lambda$ $=266 \mathrm{~nm}$ ) were used to irradiate the KDP crystal. By using appropriate shutters, single pulse selected from the train of pulses emitted for the laser or the train itself were used depending on the experiment. Time separation between pulses in the train can be changed from $0.5 \mathrm{~s}$ to $2 \mathrm{~s}$. The acoustic signal was detected by using a home-made piezoelectric transducer(PZT) (resonance at $200 \mathrm{kHz}$ ) glued directly to the crystal. Signal was registered directly in a digital oscilloscope without any further amplification. Optical energy was measured with two pyroelectric detectors. Optical measurements of decay times of the transient defects generated with UV radiation were performed using a cw $\mathrm{He}-\mathrm{Ne}(\lambda$ $=633 \mathrm{~nm}$ ) laser as a probe beam. The probe beam passed trough the irradiated crystal and the transmitted light was detected with a silicon photodiode. Transient optical spectra in the region from $\lambda=200 \mathrm{~nm}$ to $\lambda=800 \mathrm{~nm}$ was studied with a mechelle spectrograph (Mechelle 900, Multichanel Instruments, Sweden) in combination with the emission of a high-pressure Xe lamp. Since the irradiation was confined to

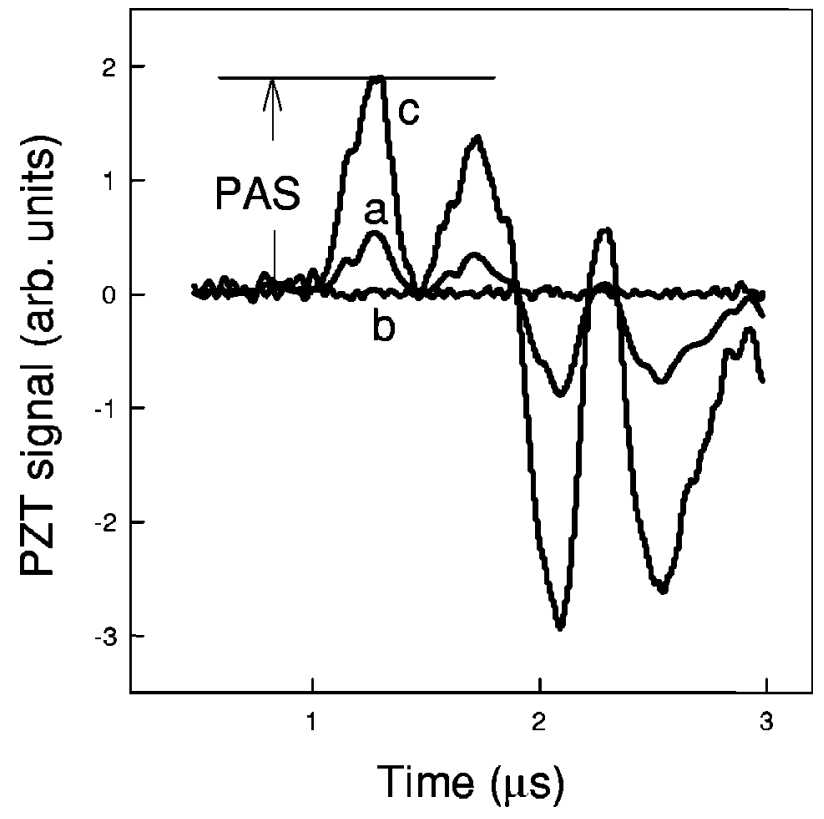

FIG. 1. Detected acoustic signals from a KDP crystal irradiated with (a) $266 \mathrm{~nm}$ pulses of $2 \mathrm{MW} / \mathrm{cm}^{2}$, (b) $532 \mathrm{~nm}$ pulses of $100 \mathrm{MW} / \mathrm{cm}^{2}$, and sample without any UV previous treatment, (c) both irradiations simultaneously.

$1 \mathrm{~mm}$ diameter spot, the spatial superposition of different beams inside the crystal was easy to obtain.

The photoacoustic signals produced in KDP after $\lambda$ $=266 \mathrm{~nm}$ or $\lambda=532 \mathrm{~nm}$ pulsed excitation were studied in terms of the following sample irradiation conditions: in some experiments the sample was treated with $\lambda=266 \mathrm{~nm}$ radiation before measurement; in other experiments the sample was free of any UV treatment. In all the experiments realized in this work, laser induced damage did not take place neither in the bulk nor in the surface of the crystal, during the radiation of the sample. If laser damage takes place in the experiment, it is possible to detect it by an increase on the photoacoustic signal when the experiments reach again the initial conditions, after photoacoustic measurements. In the experiments mentioned above we did not observe this effect.

\section{RESULTS AND DISCUSSION}

Figure 1(a) shows a typical acoustic signal detected when a KDP sample is irradiated with UV pulses $(\lambda=266 \mathrm{~nm})$ of $2 \mathrm{MW} / \mathrm{cm}^{2}\left(20 \mathrm{~mJ} / \mathrm{cm}^{2}\right)$. In this figure, it can be seen the well known ringing due to PZT resonance and some perturbations due to acoustic reflections on the surfaces of the sample. To avoid any of those effects, we measured the peak amplitude of the first oscillation (PAS in the figure) as the magnitude representative of the acoustic signal.

If the crystal is irradiated with visible light at moderate fluences, no acoustic signals were observed. The result shown in Fig. 1(b) corresponds to an experiment in which the KDP crystal was irradiated with $\lambda=532 \mathrm{~nm}$ pulses of $100 \mathrm{MW} / \mathrm{cm}^{2}\left(1 \mathrm{~J} / \mathrm{cm}^{2}\right)$. The crystal was oriented far away from phase matching angle (for second harmonic generation) 
and it was not exposed to any previous UV radiation. Figure $1(\mathrm{c})$ shows the acoustic signal detected when KDP is simultaneously irradiated with $\lambda=266 \mathrm{~nm}$ pulses with the same energy as in (a), and with $\lambda=532 \mathrm{~nm}$ pulses with the same energy as in (b). Alternatively we studied the acoustic signal generated with a green pulse of the same energy as in (b) but delayed in $0.5 \mathrm{~s}$ from the last UV pulse. The detected signal was very similar to the signal corresponding to signal (c) subtracted from signal (a). This result is against the participation of the heating of free electrons as the mechanism responsible for the acoustics signal. This conclusion is based on the fact that the lifetime of free carriers in solids is estimated in a few picoseconds.

TR defects generated for a train of UV pulses can be seen by the naked eye as a gray track in the bulk of the crystal. Absorption spectra of this zone were made; it shows absorption from UV to near infrared. The spectra is very similar to that reported in the literature, ${ }^{7-9}$ showing that the same type of species are generated. Because TR defects absorb light very efficiently at $\lambda=266 \mathrm{~nm}$ and $\lambda=532 \mathrm{~nm}$, its nonradiative relaxation must be considered in the generation of the photoacoustic signals at the conditions of this work.

Let us start with the situation corresponding to UV irradiation alone. It is accepted that the first stage is a free carrier generation by two photon absorption of UV and the stabilization of the carrier as TR defects. After that, two possible photoacoustic generation mechanisms can be considered. In the first mechanism, the TR defect is formed in an excited state. Then, the acoustic signal is due to the heat released during the rapid relaxation of these species to its ground energy state. In this situation, PAS will be independent of the amount of TR defects present in the sample. This mechanism will be called "one-step" model. The other possible mechanism assumes that photoacoustic signal is originated by a nonradiative relaxation after a linear absorption of UV radiation by the TR defects. This representation, called "twostep" model, should produce a photoacoustic signal that follows the amount of TR defects present in the sample.

In order to explore which of both models is the most appropriate to explain the origin of the acoustic signal, the following experiments were performed. In a first experiment [Fig. 2(a)], the crystal was irradiated with several UV pulses of high fluence till time zero (see irradiation scheme indicated in the inset). At this time, the energy of the pulses was reduced to one half. In this experiment the represented signal is the amplitude of the acoustical signal divided by the corresponding pulse energy. This procedure keeps away the obvious dependence of the signal with the energy of the exciting pulse and buffers the effects of laser power fluctuations. The normalized PAS signal was registered as a function of the pulse number. In Fig. 2(b) the experiment was repeated, but in this case the crystal was not previously irradiated up to $t=0 \mathrm{~s}$ (it starts free of TR defects). The crystal was excited with the same fluence as in the previous case (for $t>0$ ). In both measurements, the signal registered takes asymptotically to a constant value after nearly 30 excitation pulses.

Assuming that TR defects are generated under UV excitation, experiment (a) represents the time evolution of the

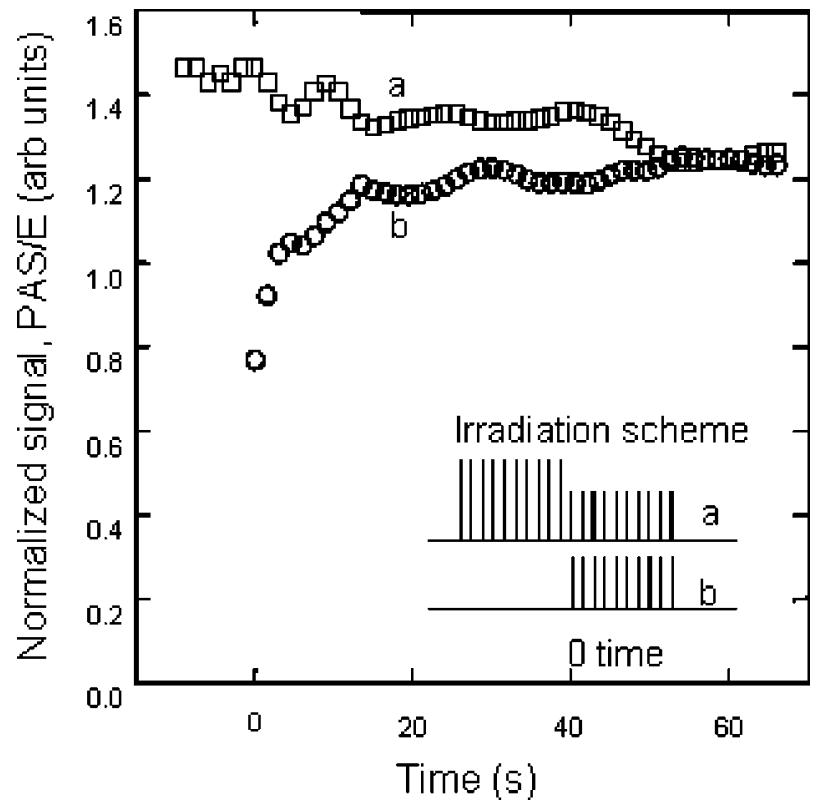

FIG. 2. Time evolution of the acoustic signal when a KDP crystal is irradiated with a train of pulses of $\lambda=266 \mathrm{~nm}$ separated each one by $1.5 \mathrm{~s}$. (a) the crystal was prepared previously by irradiation with higher energy-UV pulses, as is indicated, (b) crystal without any previous irradiation.

photoacoustic signal starting from a sample with a great number of TR defects. As time goes on after $t=0 \mathrm{~s}$, there are less of them because the UV energy has been reduced. On the other hand, experiment (b) shows the time evolution of PAS starting with a bleached crystal (without TR defects), that evolves to a situation with a stationary amount of TR defects. After the equilibrium concentration is reached, the acoustic signal remains the same in both cases. In this situation the concentration of TR defects reaches a steady-state regime: TR-defects relaxed between consecutive pulses equals the amount of TR defects generated by a single pulse. Despite the complexity of the dynamical process governing the relaxation between consecutive pulses, it is reasonable to assume that the decay time scale is several seconds. It is clear from the results of both experiments that the acoustic signal follows, at least in part, the number of TR defects as it was predicted in the two step model. But some contribution of the heating produced by the relaxation of generated TR defects (one step model) cannot be discarded.

Acoustic signals were also generated irradiating the crystal with pulses at $\lambda=532 \mathrm{~nm}$ after the sample was treated with several UV pulses in order to generate some amount of TR defects. Figure 3 shows the dependence of the photoacoustic signal generated by a single pulse at $\lambda=532 \mathrm{~nm}$ as a function of its energy. In this experiment, the crystal was previously irradiated by 15 pulses of $\lambda=266 \mathrm{~nm}$. Laser was working at $1 / 1.5 \mathrm{~Hz}$ and UV energy (single pulse energy and number of pulses) was kept constant for every measurement with the aim of generating the same amount of TR defects. PAS was measured by using $\lambda=532 \mathrm{~nm}$ pulse ( $1.5 \mathrm{~s}$ delay) after the UV radiation has been stopped (see insert in Fig. 3). As it can be seen, it is obtained a linear dependence between 


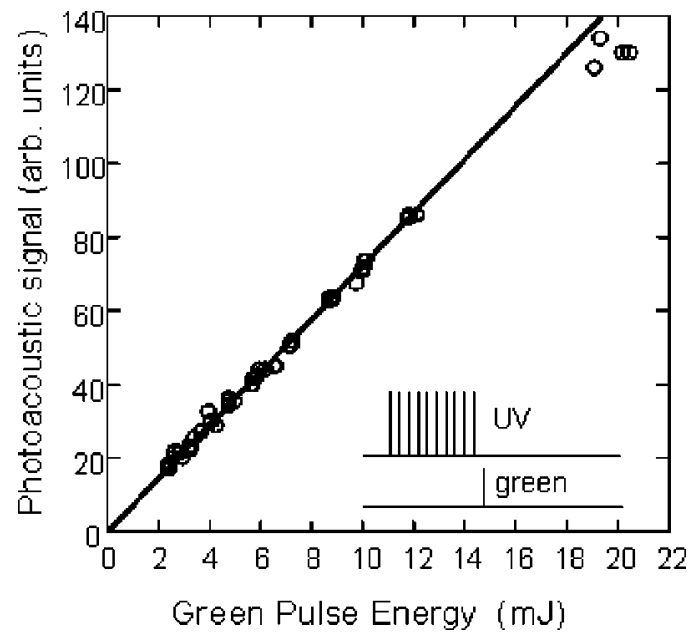

FIG. 3. Amplitude of the detected acoustic signal as a function of pulse energy of an exciting single pulse of $\lambda=532 \mathrm{~nm}$. As it is indicated in the inset the crystal was previously irradiated with several UV pulses of $\lambda=266 \mathrm{~nm}$.

the PAS signal vs the $\lambda=532 \mathrm{~nm}$ excitation energy, at least for energy level below $12 \mathrm{~mJ}$.

\section{A. Lifetime measurement of KDP transient defects}

From results of Fig. 3 and taking into account that green pulses do not generate TR defects, it can be concluded that green irradiation at $\lambda=532 \mathrm{~nm}$ only generates PAS signals by the mechanism described as the "two-step model." That is, TR defects are generated during UV radiation and are excited by green absorption. The mechanism responsible for the acoustic signals is the heat released to the medium by a nonradiative decay of the excited state of the TR defects. In addition, PAS pulses generated by green excitation below 12 $\mathrm{mJ}$ energy do not make any important reduction of the amount of TR defects. Considering these facts, we postulate that PAS is directly proportional to the TR defect concentration in the sample. So, the PAS generated by consecutive $\lambda$ $=532 \mathrm{~nm}$ pulses with energy $<12 \mathrm{~mJ}$ is used to follow the time evolution of the concentration of TR defects. The irradiation scheme was as follow: first, the sample is irradiated with enough UV pulses (rate $0.5 \mathrm{~Hz}$ ) to get a stationary concentration of TR defects and a constant photoacoustic signal; then, the sample is irradiated with green pulses $(2 \mathrm{~mJ})$ simultaneously with the UV pulses; and finally the UV is stopped and the acoustic signal registered by consecutive green pulses starts to decay as the concentration of TR defects does (Fig. 4). Time zero value for green photoacoustic signal was determined by subtracting the signal corresponding to UV from the signal corresponding to simultaneous excitation by UV plus green pulses.

Signals decay in a clearly nonexponential way and nearly $40 \mathrm{~s}$ are necessary to reduce the signal to one half of the level reached under UV irradiation. Similar results were obtained with probe green pulses of higher energy $(10 \mathrm{~mJ})$ or lower energy $(0.4 \mathrm{~mJ})$ and in experiments with different time separation between pulses $(0.1-3 \mathrm{~s})$.

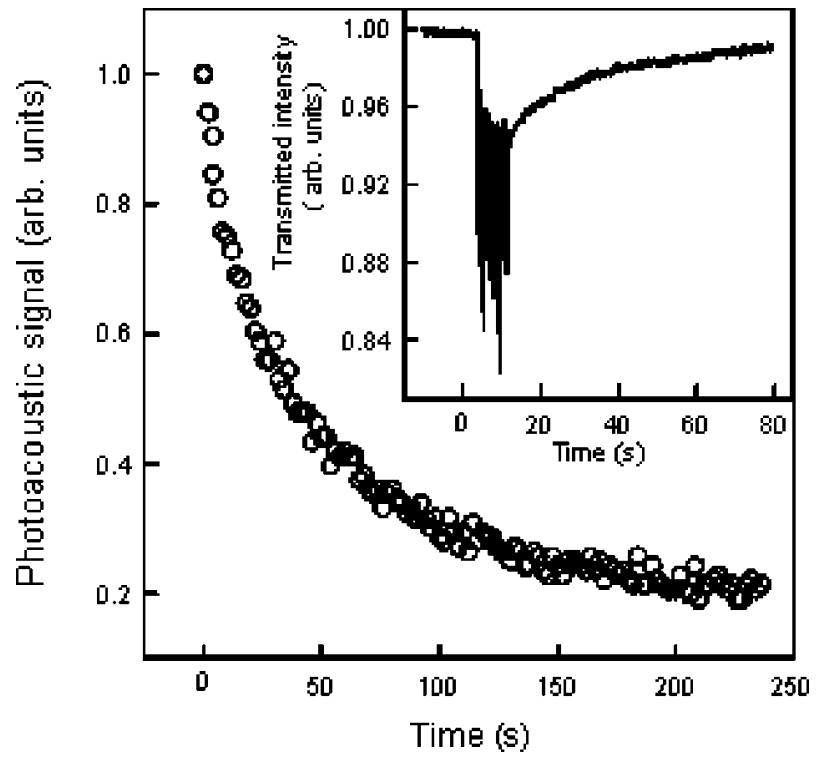

FIG. 4. Time decay of the acoustic amplitude generated by 532 $\mathrm{nm}$ pulses $(E=2 \mathrm{~mJ}$, time separation $=2 \mathrm{~s})$ in a KDP crystal. The sample was irradiated with UV pulses up to time zero; after that it was probed by the green pulses. Transient absorption of a continuum He-Ne laser at $\lambda=633 \mathrm{~nm}$ is shown in the inset; in this case UV irradiation starts at $3.7 \mathrm{~s}$ and stops at $11.3 \mathrm{~s}$.

Time-decay results of Fig. 4 can be fitted by an appropriate function that describes the time evolution of TR defects. There are several functional forms in the literature to do that, but we prefer to use the same expression used by Davis, Hughes, and Lee ${ }^{8}$ to compare our decay times with their results. Error function expression, $\mathrm{N}(\mathrm{t}) \propto \operatorname{Erf}\left[\left(\mathrm{t}_{d} / \mathrm{t}\right)^{1 / 2}\right]$, deduced from the theory developed to describe the annealing of crystalline TR defects in irradiated germanium was used. It

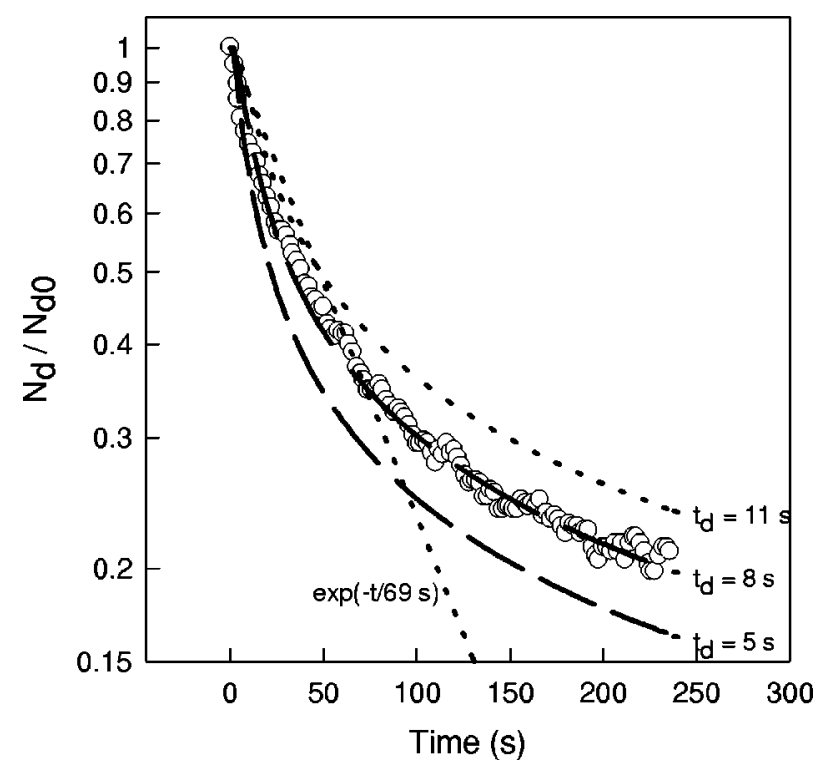

FIG. 5. Time decay of concentration of TR defects. $N_{D}$ and $N_{D 0}$ represent the concentration of TR defects at any time and in $t$ $=0 \mathrm{~s}$, respectively. Fitting function is $\operatorname{Erf}\left[\left(\mathrm{t}_{d} / \mathrm{t}\right)^{1 / 2}\right]$ as it was used in Ref. 8. 


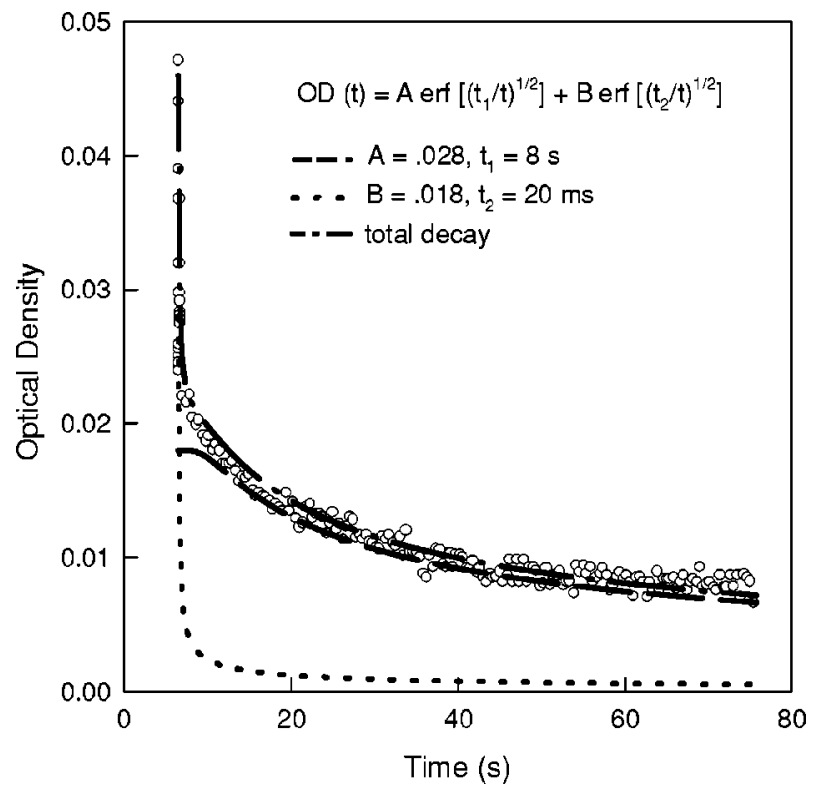

FIG. 6. Time evolution of the optical density during and after the last UV pulse (fired at $t=6.57 \mathrm{~s}$ ). Experimental points are calculated from the inset in Fig. 4. Fitting function corresponds to the superposition of two decays adjusted with error functions; amplitudes $A$ and $B$ for best fitting were 0.028 and 0.018 , respectively.

corresponds to the diffusion of a trap from a random generated position till it reaches the recombination point. Best fit was obtained for $t_{d}=(8 \pm 0.5) \mathrm{s}$. Calculated values for $\mathrm{t}_{d}$ $=11 \mathrm{~s}$ and for $t_{d}=5 \mathrm{~s}$ are also shown in Fig. 5 to remark that adjustment is more critical at longer times. For the fitting function used, the time necessary to reduce the signal to $1 / e$ is equal to $8.65 \times \mathrm{t}_{d}=69 \mathrm{~s}$. This characteristic time was used for the exponential approximation also shown in Fig. 5.

Taking into account that KDP TR defects absorb in the visible part of the spectrum, its decay time can be also determined by measuring the optical attenuation produced in $\lambda=633 \mathrm{~nm} \mathrm{cw}$ laser beam as a function of time. The inset in Fig. 4 shows the evolution of the transmitted intensity of a He-Ne laser before, during, and after UV irradiation. Transmitted beam was strongly affected at short times (several milliseconds), during and immediately after every UV pulse pump. Optical transmission measurements shown in the inset of Fig. 4 can also be fitted by appropriate function. Optical density (OD) equal to $\left[-\log \left(I_{t} / I_{i}\right)\right]$ can be calculated from measurements of transmitted intensity $\left(I_{t}\right)$ and incident intensity $\left(I_{i}\right)$. As usual, the concentration of TR-defects can be assumed proportional to OD. In Fig. 6 it is represented the decay of the OD after the UV pulses were stopped. Decay can not be fitted by one simple expression and two characteristic times seem to be involved. Fitting by two Erf are used in Fig. 6 given characteristic times of $20 \mathrm{~ms}$ and $8 \mathrm{~s}$. The characteristic time of $8 \mathrm{~s}$ is the same one found using the acoustic technique.

\section{CONCLUSIONS}

In this work we characterized the origin of the photoacoustic signals generated in KDP crystals under laser pulsed radiation. Acoustic waves are due mainly to nonradiative relaxation after linear absorption by transient TR defects. Those defects are produced by two photon absorption of UV pulses and they absorb linearly quite strongly in the visible and near UV. Then, once the TR defects are generated, irradiation with nanosecond pulses of visible light will produce high level of acoustic signals. We deduce that the most important fact of photoacoustic generation in wide band gap crystals with the property of photoinduced TR defect formation is the interaction of photons with TR defects and not the heating of free carriers during the first stage of the process. On the other hand, we use this property to measure the time decay of the generated TR defects by means of a new photoacoustic method. It consists in recording in time the evolution of the amplitude of the acoustic signals generated in the crystal by visible pulsed light. These signals are directly proportional to the amount of TR defects.

The differences found between the photoacoustic measurements and the pure optical absorption measurements in the study of the TR defects kinetics, are not completely clear. Despite the fact that we cannot work with short delays between the last UV pulse used to generate the TR defects and the first green pulse used as a probe, we do not observe an appreciable change between the population at $t=0 \mathrm{~s}$ or $t$ $=0.5 \mathrm{~s}$. This difference was less than $5 \%$. In the optical measurements we observe that more of $50 \%$ of the initial population was gone in less than $0.1 \mathrm{~s}$.

We believe that our optical experiment can be altered by thermal lensing or beam self-deformation of other origin. ${ }^{18}$ For materials such as KDP with $d n / d T<0$ (where $n$ is the refractive index and $T$ the temperature), ${ }^{20}$ the transient thermal lens is divergent. In pump-probe experiments, this fact yields a reduction in the transmitted probe intensity during and immediately after the strong pump beam. This result can be confused with a true OD if the experimental setup is not specially prepared to avoid it. This fact can be more important for low OD measurements. In our case OD was less than 0.05 while in other experiments OD's near 0.5 were reported. $^{7}$ The characteristic time for thermal lens disturbance in pulsed-laser experiments is related with the diffusivity of the sample and the radius of the spot of the heating beam. $^{21}$ For KDP, calculated values for our experimental conditions are tenths of milliseconds. Our measurement for $t_{2}$ in Fig. 6 is in this range.

Other mechanism for beam self deformation can be due to the refractive index change associated with high densities of free electrons or with the amount of TR defects itself. A reduction in the refractive index when defects are created was reported $;{ }^{9}$; the effect on transmission will be similar to a thermal lens and the OD could be overestimated. A recent work $^{22}$ shows that two kinds of TR defects can be generated in KDP crystals after UV irradiation. The first kind has a very short lifetime and it could be associated to $\mathrm{H}^{0}$ atoms or $\left(\mathrm{PO}_{4} \mathrm{H}_{2}\right)$ self-trapped hole centers. These defects could be responsible to the first stage of the OD decay measured in our experiment after UV irradiation. We believe that PAS experiments for the first millisecond of the decay time of TR 
defects can be an important tool to analyze the physical phenomenon at this relaxation time.

As it is well recognized, photoacoustic provides an excellent method to study samples with very small absorption. We show here how this advantage can be used to characterize the kinetic of TR defects generated in KDP crystals by UV excitation.

\section{ACKNOWLEDGMENTS}

We thank H. F. Ranea Sandoval for participating in many useful discussions. This work was partially financed by Projects (Grant Nos. 11X/324 and 11/I 067) of Universidad Nacional de La Plata and Project (Grant No. 03-08852) of ANPCYT.
${ }^{1}$ E. Dieguez and J.M. Cabrera, J. Phys. D 14, 91 (1981).

${ }^{2}$ E. Dieguez, J.M. Cabrera, and F. Agullo-Lopez, J. Chem. Phys. 81, 3369 (1984).

${ }^{3}$ S.D. Setzler, K.T. Stevens, L.E. Halliburton, M. Yan, N.P. Zaitseva, and J.J. Deyoreo, Phys. Rev. B 57, 2643 (1998).

${ }^{4}$ J.A. McMillan and J.M. Clemens, J. Chem. Phys. 68, 3627 (1978)

${ }^{5}$ K. Tsuchida, R. Abe, and M. Naito, J. Phys. Soc. Jpn. 35, 806 (1973).

${ }^{6}$ V.I. Salo, L.V. Atroschenko, M.I. Kolybayeva, and E.V. Scherbina, Proc. SPIE 3578, 529 (1999).

${ }^{7}$ I.N. Ogorodnikov, V. Yu Yakovlev, B.V. Shulgin, and M.K. Satybaldieva, Phys. Solid State 44, 880 (2002).

${ }^{8}$ J.E. Davis, R.S. Hughes, and H.W.H. Lee, Chem. Phys. Lett. 207, 540 (1993).

${ }^{9}$ C.D. Marshall, S.A. Payne, M.A. Henesian, J.A. Speath, and H.T. Powell, J. Opt. Soc. Am. B 11, 774 (1994).

${ }^{10}$ S.G. Demos, M. Yan, M. Staggs, J.J. De Yoreo, and H.B. Radousky, Appl. Phys. Lett. 72, 2367 (1998).

${ }^{11}$ J.O. Tocho, R. Ramirez, and J.A. Gonzalo, Appl. Phys. Lett. 59, 1684 (1991).
${ }^{12}$ D. Orzi and J.O. Tocho, J. Phys. IV 7, 245 (1994).

${ }^{13}$ M. Mesaros, O.E. Martínez, G.M. Bilmes, and J.O. Tocho, J. Appl. Phys. 81, 1 (1997).

${ }^{14}$ D.J. Orzi, N. Mingolo, G.M. Bilmes, J.O. Tocho, and O.E. Martínez, Appl. Phys. B: Lasers Opt. 66, 245 (1998).

${ }^{15}$ E. Rodríguez, J.O. Tocho, and F. Cussó, Phys. Rev. B 47, 14049 (1993).

${ }^{16}$ G.A. Torchia, J.A. Muñoz, F. Cusso, F. Jaque, and J.O. Tocho, J. Lumin. 92, 317 (2001).

${ }^{17}$ J.O. Tocho, G.M. Bilmes, and H.F. Ranea Sandoval, Appl. Phys. B: Lasers Opt. 69, 473 (1999).

${ }^{18}$ P.B. Braunlich, S.C. Jones, X. Shen, and R.T. Casper, Nucl. Instrum. Methods Phys. Res. B 46, 224 (1990).

${ }^{19}$ Lasermetrics, Inc., Teaneck, NJ 07666, USA.

${ }^{20}$ V. G. Dmitriev, G. G. Gurzadyan, and D. N. Nikogosyan, Handbook of Nonlinear Optical Crystals, 3rd ed. (Springer, Berlin, 1999), Vol. 64, p. 79.

${ }^{21}$ J.E. Tucker, C.L. Marquardt, S.R. Bowman, and B.J. Feldman, Appl. Opt. 34, 2678 (1995).

${ }^{22}$ M.M. Chirila, N.Y. Garces, L.E. Halliburton, S.G. Demos, T.A. Land, and H.B. Radousky, J. Appl. Phys. 94, 6456 (2003). 